\title{
Zur Differenzierung von Verteilungskurven klinisch-chemischer Befunde
}

\author{
Von H. Haug und D. Leupold \\ Aus der Medizinischen Universitäts-Poliklinik Wïrzburg (Direktor: Prof. Dr. H. Franke)
}

(Eingegangen am 29. Juli 1970)

Kriterien zur Beurteilung von Verteilungskurven klinisch-chemischer Analysenergebnisse unter besonderer Berücksichtigung altersabhängiger Veränderungen wurden zusammengestellt und besprochen. Eine Beurteilung der Kurvenform und von Mittelwert, häufigstem Wert und Standardabweichung eines Kollektivs von Analysenergebnissen dient der Differenzierung physiologischer und pathologischer Einflüsse im Alter. Eine Häufung von Krankheitseinflüssen drückt sich in einer Vergrößerung der Nebenkollektive, einer Zunahme der Standardabweichung und einem stärkeren Auseinanderfallen von Mittelwert und häufigstem Wert aus.

\section{The analysis of distribution curves of clinical chemical values}

Criteria of the evaluation of distribution curves of clinical chemical analyses, with spezial reference to age-dependent variations, were collected and discussed. Evaluation of the curve shape, the average value, the main concentration of frequency and the standard deviation in a collective of analytical results permits the differentiation of physiological and pathological influences in old age. An accumulation of effects due to illnes manifests itself in an enlargement of the secondary groupings, an increase in the standard deviation and a more pronounced difference between the average value and the main concentration of frequency.

Zur Beurteilung der Ergebnisse von Laboratoriumsuntersuchungen ist der Arzt auf die Kenntnis von sogenannten Normalwerten angewiesen. Die Zusammenstellung von Normalwerten erfordert die Berücksichtigung einer Reihe von Gesichtspunkten.

1. Auf die Laborbefunde wirkt sich die Fehlerbreite einer jeden Analysenmethode aus. Sie schwankt von Analyse zu Analyse und läßt sich durch systematische Fehlersuche im Laboratorium und durch die tägliche Ausführung von Standardanalysen abschätzen (1-5).

2. Der Normalbereich wird durch die biologische Streuung entscheidend beeinflußt. Es gibt bisher keine festgelegten Regeln wie man zur Erfassung eines „Normalkollektivs“ kommen kann. Die meistangewandte Untersuchung mutmaßlich gesunder junger Probanden stellt eine einseitige Auswahl dar, berücksichtigt sie doch nicht den Einfluß des Lebensalters auf die Analysenergebnisse.

Besser ist eine systematische, wahllose Erfassung der Analysenergebnisse eines ausreichend großen Querschnitts der Bevölkerung. In der Regel kann man dabei erwarten, zwei oder mehr Kollektive zu finden:

1. ein großes Kollektiv von Probanden mit „normalen“ Laborbefunden,

2. ein oder mehrere Kollektive mit davon abweichenden Befunden.

Bei Probanden, die sich völlig gesund fühlen, können subklinische Erkrankungen zu einer Verfälschung der „Normalwerte“ führen. Diese Grenzbefunde stellen ein großes Problem dar, da die Übergänge zwischen "normalen" und pathologischen Werten fließend sind. Wir haben an einem großen poliklinischen Krankengut eine Zusammenstellung von Laborergebnissen unter besonderer Berücksichtigung des Lebensalters vorge- nommen und teilen hier einige Überlegungen mit, die zur Differenzierung von Verteilungskurven verschiedener Altersklassen hilfreich sind.

\section{Verteilungskurven, die mehrere Kollektive um- fassen}

Ist ein Kollektiv nicht normal verteilt, so sind grundsätzlich drei Möglichkeiten in Betracht zu ziehen (6):

1. Das Kollektiv ist nicht homogen, es ist $z$. B. aus zwei normal verteilten Kollektiven zusammmengesetzt. Dadurch erklären sich schräge, gebuckelte und mehrgipflige Kurven.

2. Das an sich normal verteilte Kollektiv wurde nicht in dem ihm gemäßen Maßstab gemessen. Eine Änderung des Abszissenmaßstabes z. B. durch logarithmische Transformation wird in solchen Fällen zur Normalisierung führen (lognormale Verteilung).

3. Das Kollektiv ist tatsächlich nicht normal verteilt und läßt sich weder durch Änderung der Auswahlbedingungen noch durch Änderung des Maßstabes normalisieren.

Da ein zufälliges Beobachtungsgut auch Probanden mit pathologischen Laborwerten enthält, dürfen wir folgenden Verteilungstyp erwarten: Das Normalkollektiv wird von je einem Kollektiv von Patienten mit pathologisch hohen bzw. pathologisch niedrigen Werten eingeschlossen (Abb. 1). In der Praxis sind die Verhältnisse noch komplizierter, da es neben dem Normalkollektiv zahlreiche weitere Kollektive geben kann, die sich mehr oder weniger überlappen. In vielen Fällen muß bei einer mehrgipfligen Kurve auch an einen genetischen Polymorphismus gedacht werden. Genetisch bedingt und pathologisch bedingt hohe oder niedere Unterkollektive sind oft nicht zu trennen. Auf dem Gebiet 
der klinischen Chemie sind unsere Kenntnisse über genetische Einflüsse aber noch dürftig.

Es ist allgemein üblich, den Bereich normaler Werte auf Grund der Häufigkeitsverteilung der Werte ,normaler", gesunder Probanden zu definieren. Gewöhnlich wird der Mittelwert $\pm 2 \mathrm{~s}$ als Normalbereich angegeben. Werte außerhalb des Normalbereichs sind auf einen pathologischen Prozeß verdächtig. Gerade deshalb exscheint es uns notwendig, nicht nur die Verteilung der Werte unter den gesunden, sondern auch unter denjenigen kranken Probanden zu kennen, deren Krankheit mit einer Veränderung des untersuchten Merkmals einhergeht. Um eine übersichtliche Darstellung zu erzielen, wurde im folgenden dem Normalkollektiv nur ein Kollektiv mit pathologisch hohen Werten gegenübergestellt.

Nach Koller (7) ergeben sich drei grundsätzlich verschiedene Lagemöglichkeiten zwischen diesen beiden Kollektiven (Abb. 2).

Im Fall A können alle Werte innerhalb der doppelten Standardabweichung des Normalkollektivs als normal

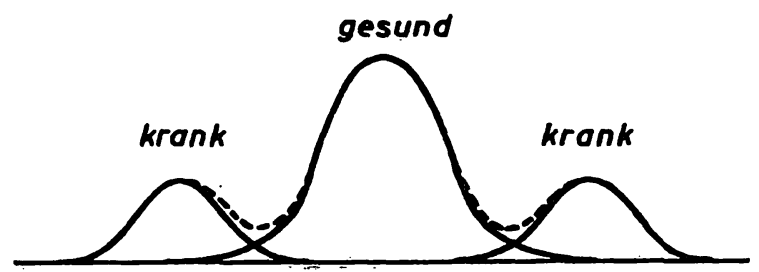

Abb. 1

Häufigkeitsverteilung, die bei der Zusammenstellung von Laborbefunden eines unausgesuchten Kollektivs in der Regel zu erwarten sind
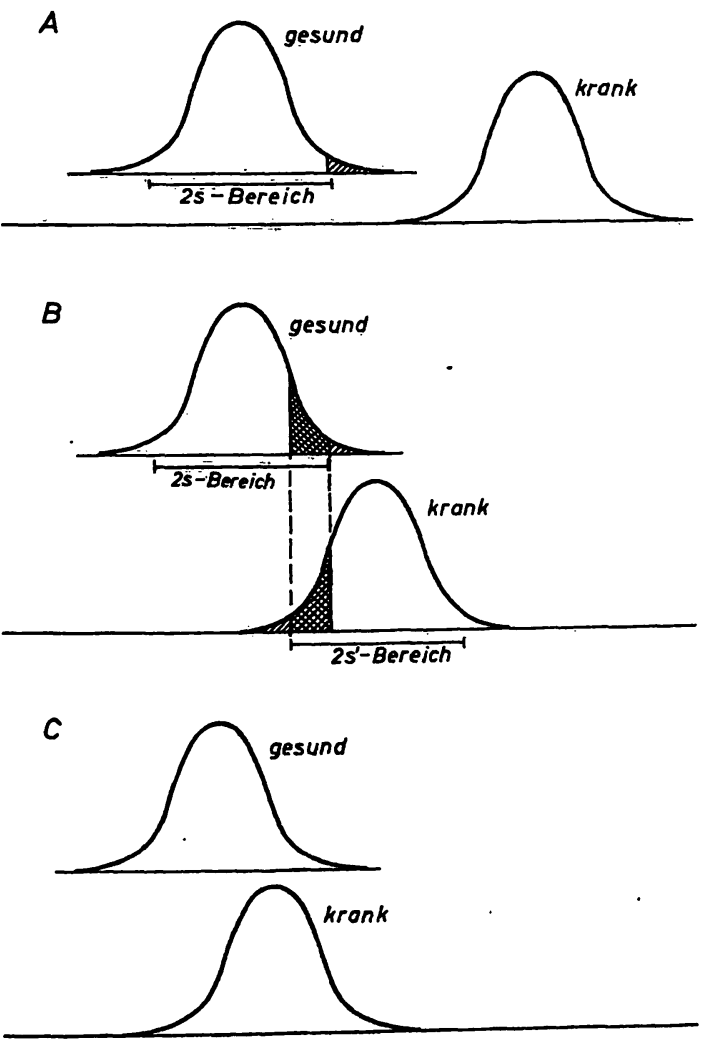

Abb. 2

Verschiedene Lagemöglichkeiten eines "gesunden" und gleichgroßen „kranken" Kollektivs zueinander (KOLLER (7)) angesehen werden. Die Werte der kranken Probanden liegen dann außerhalb des angegebenen Normalbereiches.

Würde man im Fall B ebenso nur die Häufigkeitsverteilung der gesunden Probanden zur Ermittlung des Normalbereiches heranziehen, so müßte man einen beträchtlichen Teil des „kranken" Kollektives als gesund ansehen (karierte Fläche). Nach Koller sollte man deshalb vom $2 \mathrm{~s}$ Bereich des "kranken" Kollektivs ausgehen, muß dann aber in Kauf nehmen, daß ein Teil des "gesunden" Kollektivs als krank angesehen wird (karierte Fläche im "gesunden" Kollektiv). Dieser Randeffekt ist bei dem angegebenen Verfahren unvermeidlich. Im Fall $C$ überlappen sich die beiden Verteilungskurven weitgehend. Selbst wenn eine signifikante Differenz zwischen den Mittelwerten beider Kollektive besteht, kann kein vernünftiger Normalbereich angegeben werden.

Wichtig für die Angabe der Normalbereiche ist die Mitteilung der Standardabweichung (s). Je nach Strenge der Kriterien wird in der Literatur entweder der $1 \mathrm{~s}-$ Bereich, 2 s-Bereich oder $3 \mathrm{~s}$-Bereich angegeben. In der Regel wird nur ein Bereich genannt, innerhalb dessen Grenzen die Probanden gesund und außerhalb dessen Grenzen die Patienten krank sein sollen. Diese Schwarzweißmalerei ist für den beurteilenden Arzt recht bequem, aber nach unserer Meinung doch nicht korrekt; die Übergänge zwischen gesund und krank sind fließend. Für unsere Normalwerte möchten wir deshalb nach den Empfehlungen von Rost (8) zwei Bereiche angeben:

1. den $1 \mathrm{~s}$-Bereich; Probanden mit Werten innerhalb dieser Grenzen sind wahrscheinlich gesund.

2. den 2s-Bereich; Werte, die zwischen $1 \mathrm{~s}$ und $2 \mathrm{~s}$ liegen, sind möglicherweise pathologisch, können aber auch "gesund" sein.

3. Die Werte jenseits des $2 \mathrm{~s}$-Bereichs sind wahrscheinlich pathologisch.

Unser Beobachtungsgut läßt sich demnach in ein Kollektiv wahrscheinlich gesunder Probanden, in Probanden mit fraglichen Befunden und in Probanden mit wahrscheinlich pathologischen Befunden unterteilen. Wenn sich die Kollektive Gesunder und Kranker nach Abbildung 2 Fall $\mathrm{A}$ verteilen, ist diese Definition zu streng. Diese Schärfe der Trennung dürfte aber im biologischen Bereich selten vorkommen. Der Normalbereich kann dann auch mit $\bar{x} \pm 2 s$, der Verdachtsbereich mit $\bar{x} \pm 3 s-$ $\overline{\mathrm{x}} \pm 2 \mathrm{~s}$ angesetzt werden. Diese Kriterien müssen für jede Untersuchungsmethode festgelegt werden.

Unterschiede in den Verteilungskurven als Ausdruck altersabhängiger Veränderungen

Bisher wird das Lebensalter bei Normalwertangaben selten berücksichtigt. Als Beispiel einer altersabhängigen Veränderung wählen wir ein häufigeres Auftreten höherer Laborwerte mit fortschreitendem Alter. Der damit verbundene Anstieg der Mittelwerte kann verschiedene Ursachen haben. Entweder hat sich das 
gesamte normale Kollektiv durch physiologische Altersveränderungen $\mathrm{zu}$ höheren Werten hin verschoben (Abb. 3 Fall A), oder das Kollektiv mit pathologischen Werten hat relativ zugenommen; es wurden häufiger kranke Probanden erfaßt (Abb. 3 Fall B). Schließlich können auch beide Veränderungen nebeneinander ablaufen (Abb. 3 Fall C).

Der in der statistischen Auswertung durch Mittelwert und doppelte Standardabweichung festgelegte Bereich verschiebt sich im Alter sowohl im Fall $A$ als auch im Fall B zu höheren Werten hin. Wie aus der graphischen Darstellung ersichtlich, erscheint aber eine Änderung des Normal-Bereichs nur im Fall A sinnvoll. Trotz eines Anstiegs des arithmetischen Mittels bleibt. im Fall $B$ der Normalbereich konstant.

Das größere linke Kollektiv entspreche den gesunden Probanden. Die Trennung der Kollektive wird häufig nicht so gut $T$ wie hier aus didaktischen Gründen gekennzeichnet - erkennbar sein. Die Möglichkeit einer zusätzlichen Verschiebung des Kollektivs pathologischer Werte wurde bei diesen Überlegungen aus Gründen der Übersichtlichkeit außer acht gelassen.

Einen weiteren guten Anhalt zur Beurteilung der Frage, ob eine altersbedingte Veränderung vorliegt oder nicht, bietet der häufigste oder dichteste Wert einer Verteilungskurve. Verschiebt sich der häufigste Wert, der nicht gleich dem Mittelwert sein muß, und mit ihm das Hauptkollektiv (Fall A und C s. Abb. 3), so sehen wir diese Veränderung eher als physiologisch an. Denn es ist
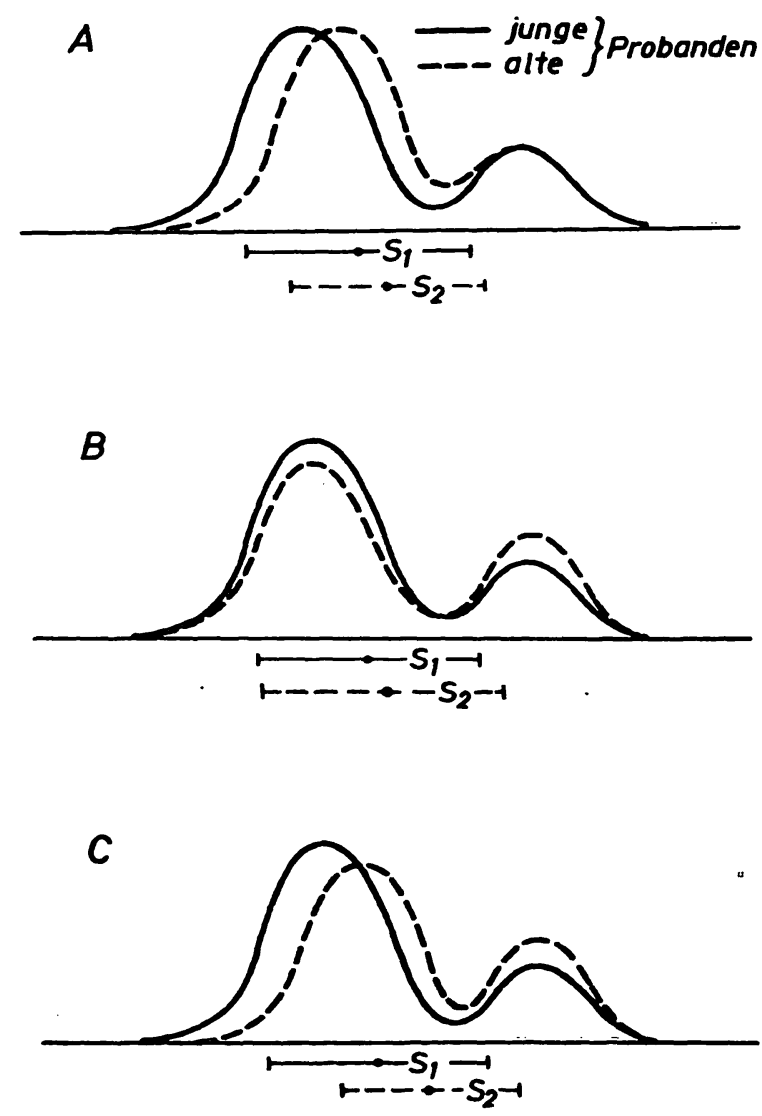

Abb. 3 Mögliche Veränderungen der Häufigkeitsverteilungen als Ausdruck
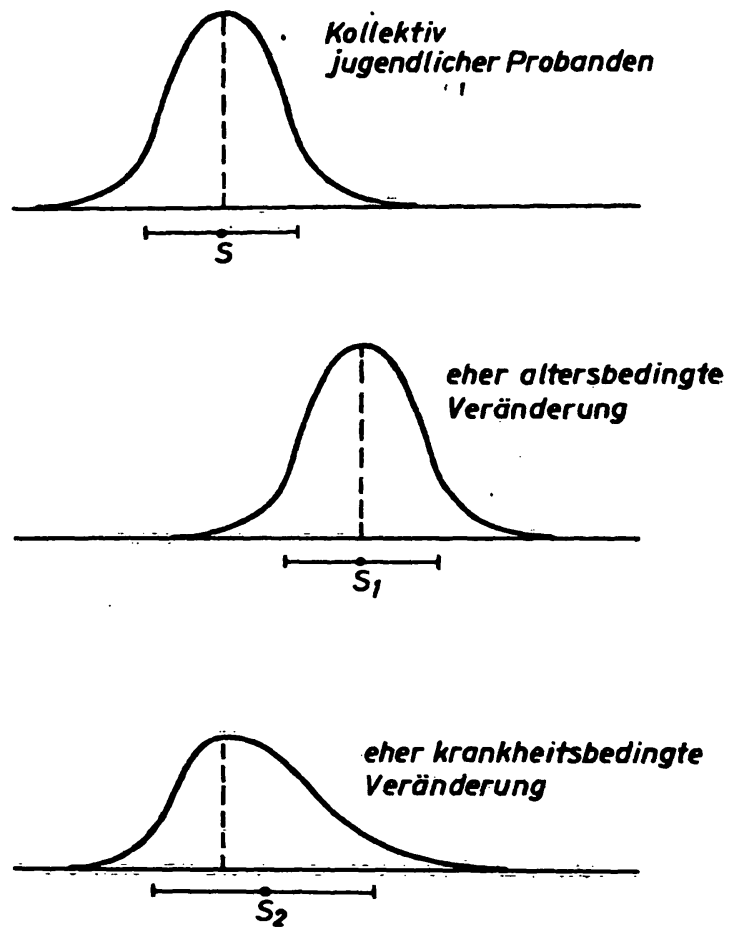

Abb. 4

Veränderung der Standardabweichung als Kriterium für eine wahrscheinlich krankheitsbedingte oder wahrscheinlich altersbedingte Veränderung der Laborwerte

unwahrscheinlich, daß der Großteil der untersuchten Probanden insgesamt im Alter pathologische Werte aufweist. Dies gilt um so mehr, wenn die Veränderung der Analysenergebnisse in hohem $\mathrm{Maße}$ an eine Erkrankung eines Organs gebunden ist. Es ist besonders unwahrscheinlich, daß alle alten Menschen an der gleichen Organerkrankung leiden. Ist dagegen der dichteste Wert mit fortschreitendem Alter unverändert geblieben, so spricht eine Verschiebung des Mittelwertes. dafür, $\mathrm{da} ß$ häufig kranke Probanden erfaßt wurden. Nimmt ferner die Standardabweichung mit fortschreitendem Alter zu, so müssen auftretende Verschiebungen der Kollektive eher als krankheitsbedingt angesehen werden. Bleibt dagegen die Standardabweichung annähernd gleich groß, so macht dies eine altersbedingte Veränderung wahrscheinlich (vgl. Abb. 3 und 4).

Die von uns zusammengetragenen und aufgestellten Kriterien haben sich bei der Beurteilung von Verteilungskurven, bei denen neben Gesunden auch mehr oder weniger kranke Probanden erfaßt werden, als nützlich erwiesen.

\section{Literatur}

1. Bürtner, H., diese Z. 3, 69 (1965). - 2. BürtNer, H. und D. Stamm, diese Z. 4, 303 (1966). - 3. BürtrNer, H., diese Z. 5 , 41 (1967). - 4. Büttner, H., diese Z. 7, 89 (1968). - 5. Stamm, D. und H. BürTNER, diese Z. 7, 393 (1969). - 6. Documenta Geigy, Wissenschaftliche Tabellen. 6. Aufl., J. R. Geigy A: G. Basel (1955). - 7. Koller, S. Bibl. haemat. 21, 125 (1965). 8. Rost, G., G. HonigmanN und V. Schliak: Z. ges. inn. Med. 21 289 (1966).

Priv. Doz. Dr. H. Haug 87 Würzburg Klinikstr. 8 